\title{
Error-resilient Floquet geometric quantum computation
}

\author{
Yuan-Sheng Wang $\odot,{ }^{1,2}$ Bao-Jie Liu $\odot,{ }^{1}$ Shi-Lei Su, ${ }^{3}$ and Man-Hong Yung ${ }^{1,4,5,6, *}$ \\ ${ }^{1}$ Department of Physics, Southern University of Science and Technology, Shenzhen 518055, China \\ ${ }^{2}$ School of Physical Sciences, University of Science and Technology of China, Hefei 230026, China \\ ${ }^{3}$ School of Physics, Zhengzhou University, Zhengzhou 450001, China \\ ${ }^{4}$ Shenzhen Institute for Quantum Science and Engineering, Southern University of Science and Technology, Shenzhen 518055, China \\ ${ }^{5}$ Guangdong Provincial Key Laboratory of Quantum Science and Engineering, Southern University of Science and Technology, \\ Shenzhen 518055, China \\ ${ }^{6}$ Shenzhen Key Laboratory of Quantum Science and Engineering, Southern University of Science and Technology, Shenzhen 518055, China
}

(Received 21 September 2020; accepted 7 June 2021; published 2 July 2021)

\begin{abstract}
We propose a geometric quantum computation (GQC) scheme, called Floquet GQC (FGQC), where errorresilient geometric gates based on periodically driven two-level systems can be constructed via a non-Abelian geometric phase proposed in a recent study [V. Novičenko and G. Juzeliūnas, Phys. Rev. A 100, 012127 (2019)]. Based on Rydberg atoms, we give possible implementations of universal FGQC single-qubit gates and a nontrivial FGQC two-qubit gate. By using numerical simulation, we evaluate the performance of the FGQC Z and $\mathrm{X}$ gates in the presence of both decoherence and a certain kind of systematic control error. For the currently available coherence time of the Rydberg state, $T_{2} \approx 32 \mu \mathrm{s}$, the numerical results show that the $\mathrm{X}$ and $\mathrm{Z}$ gate fidelities are about 0.900 and 0.899 , respectively. In addition, we find that FGQC is robust against global control error; both analytical demonstration and numerical evidence are given. As the coherence time of various qubits grows, FGQC may provide a promising error-resilient quantum computation scheme in the future.
\end{abstract}

DOI: 10.1103/PhysRevResearch.3.033010

\section{INTRODUCTION}

Quantum computations can solve certain problems much more effectively than classical computations, such as quantum simulations [1,2], prime factoring [3-5], searching unsorted data [6], and machine learning [7-9]. High-fidelity universal gates for quantum bits form an essential ingredient of quantum computation (QC) and quantum information processing. One promising approach towards this goal is to use geometric phases [10-13] that have attracted much attention because geometric phases depend only on the global properties of the evolution trajectories, and thus have built-in noise-resilient features against certain local noises [14-18].

The early applications of geometric quantum computation (GQC) depend on adiabatic quantum evolution to suppress transitions between different instantaneous eigenstates of the Hamiltonian [19-23]. These adiabatic gates operate slowly compared to the dynamical time scale. They become vulnerable to decoherence effects that may lead to the loss of coherence. To overcome the dilemma between the limited coherence times and the long duration of adiabatic evolution, nonadiabatic geometric quantum computation

\footnotetext{
*yung@ sustech.edu.cn

Published by the American Physical Society under the terms of the Creative Commons Attribution 4.0 International license. Further distribution of this work must maintain attribution to the author(s) and the published article's title, journal citation, and DOI.
}

(NGQC) [24-30] and nonadiabatic holonomic quantum computation (NHQC) [31-44] based on nonadiabatic Abelian and non-Abelian geometric gates [11,13] have been proposed. Owing to its intrinsic noise-resistance features and high-speed implementation, NGQC has attracted considerable interest [27,31,34,45-71] and has been experimentally demonstrated with nuclear magnetic resonance [45,46], superconducting circuits [47-51,72-74], and nitrogen-vacancy centers in diamond [52-57]. However, these nonadiabatic gates require the driving pulses to satisfy the restrictive conditions, thus reducing the robustness of the resulting geometric gates against control errors [41-44]. On the other hand, the use of periodic control pulses has emerged as a ubiquitous tool for the coherent control and engineering of quantum dynamics [75-81], with the recent increase in applications in constructing GQC scenarios [82-85].

In this paper, we propose a geometric computation scheme, called Floquet GQC (FGQC), where universal error-resistant geometric gates can be constructed via a non-Abelian geometric phase. This non-Abelian geometric phase emerges from a periodically driven quantum system and was found in a recent study [83]. Based on Rydberg atoms, a possible experimental realization of our proposal is provided. By applying this proposal, in the presence of both decoherence and a certain kind of systematic control error, numerical simulations of the $\mathrm{Z}$ and $\mathrm{X}$ gates are given. The numerical results show that, at present, the performance of FGQC based on the Rydberg state is limited by the short coherence time of the latter. Furthermore, we find that FGQC is robust against global control error. An analytical demonstration is given. 
In addition, using a possible set of parameters, we simulate FGQC X, Z, and nontrivial two-qubit gates in the presence of global control error, then we compare their performances with those of the same gates based on dynamical gate (DG) and NGQC scenarios [24-30]; the results are shown in Fig. 3. These numerical results provide evidence of the superiority of FGQC gates over NGQC and standard DG gates, in terms of solving global control error.

This paper is organized as follows. In Sec. II, the general theory of GQC and FGQC is introduced. In Sec. III, a possible implementation of universal single-qubit and nontrivial two-qubit gates using Rydberg atoms is extensively studied concretely based on the proposed theory in Sec. II. Section IV summarizes the findings.

\section{GENERAL THEORY}

\section{A. General theory of GQC}

Consider a quantum system exposed to Hamiltonian $H(t)$. For any set of complete basis vectors $\left\{\left|\psi_{\alpha}(0)\right\rangle\right\}$ at $t=0$, the unitary temporal evolution operator can be expressed as $U(t, 0)=\mathcal{T} e^{-i \int_{0}^{t^{\prime}} H\left(t^{\prime}\right) d t^{\prime}}=\sum_{\alpha}\left|\psi_{\alpha}(t)\right\rangle\left\langle\psi_{\alpha}(0)\right|$, where $\mathcal{T}$ is the time-ordering operator and $\left|\psi_{\alpha}(t)\right\rangle=$ $\mathcal{T} e^{-i \int_{0}^{t^{\prime}} H\left(t^{\prime}\right) d t^{\prime}}\left|\psi_{\alpha}(0)\right\rangle$ is a time-dependent state satisfying the Schrödinger equation. Now, at each moment of time, a different set of time-dependent bases can be selected, $\left\{\left|\mu_{\alpha}(t)\right\rangle\right\}$, satisfying the boundary conditions at $t=0$ and $t=\tau$ :

$$
\left|\mu_{\alpha}(\tau)\right\rangle=\left|\mu_{\alpha}(0)\right\rangle=\left|\psi_{\alpha}(0)\right\rangle .
$$

The time-evolution state can be written as $\left|\psi_{\alpha}(t)\right\rangle=$ $\sum_{\beta} c_{\alpha \beta}(t)\left|\mu_{\beta}(t)\right\rangle$. By substituting this equation into the Schrödinger equation, we have

$$
\frac{d}{d t} c_{\alpha \beta}(t)=i \sum_{\gamma}\left[A_{\alpha \gamma}(t)-H_{\alpha \gamma}(t)\right] c_{\gamma \beta}(t),
$$

where $\quad H_{\alpha \beta}(t)=\left\langle\mu_{\alpha}(t)|H(t)| \mu_{\beta}(t)\right\rangle \quad$ and $\quad A_{\alpha \beta}(t)=$ $\left\langle\mu_{\alpha}(t)\left|i \partial_{t}\right| \mu_{\beta}(t)\right\rangle \mid$, which can be combined to form an effective Hamiltonian: $H_{\mathrm{eff}}(t)=R^{\dagger}(t)[H(t)-i d / d t] R(t)$, with $R(t)=\sum_{\alpha}\left|\mu_{\alpha}(t)\right\rangle\left\langle\mu_{\alpha}(0)\right|$. Consider any unitary once differentiable operator $V(t)$ satisfying the boundary condition $V(\tau)=V(0)$. The operator $A(t)$ is transformed as a proper gauge potential under the basis change, $\left|\mu_{\alpha}(t)\right\rangle \rightarrow \sum_{\gamma}\left|\mu_{\alpha}(t)\right\rangle V_{\alpha \gamma}(t), \quad$ and $A(t)$ will generally contribute a non-Abelian geometric phase to the temporal evolution operator $U(\tau, 0)$.

For a GQC scenario, the dynamical phase should be eliminated by engineering $H(t)$ and the ancillary basis $\left\{\left|\mu_{\alpha}(t)\right\rangle\right\}$. If the effective Hamiltonian is always diagonal in the initial basis, $A_{\alpha \beta}(t)-H_{\alpha \beta}(t)=\delta_{\alpha \beta}\left[A_{\alpha \alpha}(t)-H_{\alpha \alpha}(t)\right]$. Then, the time-evolution operator can be written as follows:

$$
U(t, 0)=\sum_{\alpha} e^{i \phi_{\alpha}(t)}\left|\mu_{\alpha}(t)\right\rangle\left\langle\mu_{\alpha}(0)\right|
$$

where $\phi_{\alpha}(t)=\int_{0}^{t} d t^{\prime}\left[A_{\alpha \alpha}\left(t^{\prime}\right)-H_{\alpha \alpha}\left(t^{\prime}\right)\right]$ is the sum of the geometric and dynamical phases. In NHQC [32,33], parallel transport conditions $\left\langle\mu_{\alpha}(t)|H(t)| \mu_{\alpha}(t)\right\rangle=0$ were applied to the Hamiltonian to remove the dynamical phases, while in the $\mathrm{NHQC}+[31]$ scenario the parallel transport condition was replaced with a wilder condition $\int_{0}^{\tau} d t\left\langle\mu_{\alpha}(t)|H(t)| \mu_{\alpha}(t)\right\rangle=$ 0 at the end of the cyclic evolution, making it possible for NHQC + to be compatible with most of the optimization schemes.

\section{B. Offsetting the dynamical phase using a part of the geometric phase}

In this subsection, another way to implement GQC is introduced, where the dynamical phase is offset by a part of the geometric phases [86].

Consider a set of ancillary states $\left\{\left|\mu_{\alpha}(t)\right\rangle\right\}$ dependent on two time-varying real parameters: $\lambda_{1}(t)$ and $\lambda_{2}(t)$. Then, $\left|\mu_{\alpha}(t)\right\rangle=R(\lambda)\left|\mu_{\alpha}(0)\right\rangle$ and $A_{\alpha \beta}(t)=A_{\alpha \beta}^{\left(\lambda_{1}\right)}(t)+$ $A_{\alpha \beta}^{\left(\lambda_{2}\right)}(t)$, where $\lambda(t)=\left(\lambda_{1}(t), \lambda_{2}(t)\right)^{\mathrm{T}}$ and

$$
A_{\alpha \beta}^{\left(\lambda_{j}\right)}(t)=\dot{\lambda}_{j}(t)\left\langle\mu_{\alpha}(\lambda)\left|i \frac{\partial}{\partial \lambda_{j}}\right| \mu_{\beta}(\lambda)\right\rangle,
$$

that is, the gauge potential can be divided into two parts: $A^{\left(\lambda_{1}\right)}(t)$ and $A^{\left(\lambda_{2}\right)}(t)$. If $A^{\left(\lambda_{1}\right)}(t)$ is equal to the dynamical part of the effective Hamiltonian, $A_{\alpha \beta}^{\left(\lambda_{1}\right)}(t)=H_{\alpha \beta}(t)$, then the timeevolution operator is governed by a part of gauge potential $A_{\alpha \beta}^{\left(\lambda_{2}\right)}(t)=A_{\alpha \beta}(t)-H_{\alpha \beta}(t)$. For example, consider $R(\lambda)=$ $e^{X(\lambda)} ;$ then

$$
A_{\alpha \beta}^{\left(\lambda_{1}\right)}(t)=\left\langle\mu_{\alpha}(\lambda)\left|\left[i \dot{\lambda}_{1}(t) \frac{\partial X(\lambda)}{\partial \lambda_{1}}\right]\right| \mu_{\beta}(\lambda)\right\rangle .
$$

By substituting $H(t)=i \dot{\lambda}_{1}(t) \frac{\partial X(\lambda)}{\partial \lambda_{1}}$ in the effective Hamiltonian $H_{\mathrm{eff}}(t), H_{\alpha \beta}(t)$ will be canceled out with $A_{\alpha \beta}^{\left(\lambda_{1}\right)}(t)$. The operator $A^{\left(\lambda_{2}\right)}(t)$ is transformed as a gauge potential under the change $\left|\mu_{\alpha}(t)\right\rangle \rightarrow \sum_{\beta}\left|\mu_{\alpha}(t)\right\rangle V_{\alpha \beta}^{\prime}\left(\lambda_{2}\right)$, where $V^{\prime}\left[\lambda_{2}(t)\right]$ is any once differentiable operator satisfied $V^{\prime}\left[\lambda_{2}(\tau)\right]=V^{\prime}\left[\lambda_{2}(0)\right]$.

For a more specific example, consider $X(t)$ in the following form:

$$
X[\lambda(t)]=-i F\left[\lambda_{1}(t)\right] H_{0}\left[\lambda_{2}(t)\right],
$$

where $F\left(\lambda_{1}\right)$ is a $\lambda_{1}$-dependent real function and $H_{0}\left(\lambda_{2}\right)$ is a $\lambda_{2}$-dependent hermitian operator. To cancel out the dynamical part of the effective Hamiltonian, $H(t)=i \dot{\lambda}_{1}(t) \frac{\partial X}{\partial \lambda_{1}}=$ $\dot{\lambda}_{1}(t) \frac{\partial F\left(\lambda_{1}\right)}{\partial \lambda_{1}} H_{0}\left(\lambda_{2}\right)$ is selected, resulting in a pure geometric effective Hamiltonian $H_{\text {eff }}(t)=A^{\left(\lambda_{2}\right)}(t)=\dot{\lambda}_{2}(t) R^{\dagger}(\lambda) i \partial_{\lambda_{2}} R(\lambda)$. Then, Eq. (2) can be simplified to

$$
\frac{d}{d t} c_{\alpha \beta}(t)=i \sum_{\gamma} A_{\alpha \gamma}^{\left(\lambda_{2}\right)}(t) c_{\gamma \beta}(t) .
$$

The formal solution of Eq. (7) can be expressed as follows: $c(t)=\mathcal{T} \exp \left[i \int_{0}^{t} A^{\left(\lambda_{2}\right)}\left(t^{\prime}\right) d t^{\prime}\right]$. Using the cyclic condition $\left|\mu_{\alpha}(\tau)\right\rangle=\left|\mu_{\alpha}(0)\right\rangle$ and the definition of $c_{\alpha \beta}(t),\left|\psi_{\alpha}(\tau)\right\rangle=$ $\sum_{\beta} c_{\alpha \beta}(\tau)\left|\mu_{\beta}(0)\right\rangle$, indicating that $c(\tau)$ is just the transformation matrix from the initial states to the final states. Therefore, the temporal evolution operator can be expressed as follows:

$$
U(\tau, 0)=c(\tau)=\mathcal{T} e^{i \int_{0}^{t} A^{\left(\lambda_{2}\right)}\left(t^{\prime}\right) d t^{\prime}} .
$$

This cyclic unitary time-evolution operator is geometric because $A^{\left(\lambda_{2}\right)}(t)$ is a gauge potential. 


\section{Non-Abelian geometric phases in periodically driven systems}

In this subsection, a brief introduction of the work reported in Ref. [83] is provided, demonstrating that a non-Abelian geometric phase will form in the adiabatic evolution of a quantum system within a fully degenerate Floquet band.

Consider a driven quantum system with the following Hamiltonian:

$$
H(t)=f\left[\lambda_{1}(t)\right] H_{0}\left[\lambda_{2}(t)\right]
$$

where $f\left(\lambda_{1}+T\right)=f\left(\lambda_{1}\right)$ is a periodic real function with pe$\operatorname{riod} T$ and $\int_{0}^{T} f\left(\lambda_{1}\right) d \lambda_{1}=0$. To remove the dynamical phase using the strategy introduced in Sec. II B, $R(\lambda)=e^{X(\lambda)}$ should be selected, where $X(\lambda)$ can be expressed as (6). $F\left(\lambda_{1}\right)$ is the primitive function of $f\left(\lambda_{1}\right)$. It can be verified that $F\left(\lambda_{1}\right)$ is also periodic with period $T$, as are both $R(\lambda)$ and $A^{\left(\lambda_{2}\right)}\left(\lambda_{1}, t\right)$. $A^{\left(\lambda_{2}\right)}\left(\lambda_{1}, t\right)$ and $c\left(\lambda_{1}, t\right)$ can be expanded in a complete set of bases of $\lambda_{1}$ parameter space, $\left\{e^{i l \lambda_{1}(2 \pi / T)} \mid l \in Z\right\}$, with which Eq. (7) can be recast into

$$
\frac{d}{d t} c_{\alpha \beta}^{(k)}(t)=\sum_{\gamma ; m} i H_{\alpha \gamma ; k m}^{F}(t) c_{\gamma \beta}^{(m)}(t),
$$

where $\quad H^{F}(t) \equiv A^{\left(\lambda_{2}\right)}\left(\lambda_{1}, t\right)-i \partial_{\lambda_{1}} \quad$ and $\quad O_{\alpha \gamma ; k m}(t)=$ $(1 / T) \int_{0}^{T} d \lambda_{1} e^{-i(k-m)(2 \pi / T) \lambda_{1}}\left\langle\mu_{\alpha}(\lambda)|O(t)| \mu_{\gamma}(\lambda)\right\rangle \quad$ is the matrix element of operator $O(t)$. When $\lambda_{1}(t)=\omega t$ and $T=2 \pi$, we have

$$
H_{\alpha \gamma ; k m}^{F}(t)=A_{\alpha \gamma ; k m}^{\left(\lambda_{2}\right)}(t)-k \omega \delta_{k m} \delta_{\alpha \gamma} .
$$

Clearly, $|\tilde{l}\rangle=e^{i l(2 \pi / T) \lambda_{1}}$ are the eigenbases of operator $i \partial_{\lambda_{1}}$ with eigenvalues $-l(2 \pi / T)$. These eigenvalues do not change when the time $t$ is varied; hence, they form bands, which are referred to as Floquet bands [83]. In other words, the operator $i \partial_{\lambda_{1}}$ is diagonal in basis $|\tilde{l}\rangle$. The operator $A^{\left(\lambda_{2}\right)}\left(\lambda_{1}, t\right)$ in general is not diagonal in this basis; its off-diagonal terms describe the coupling between different Floquet bands. However, according to Eq. (4), $A_{\alpha \gamma ; k m}^{\left(\lambda_{2}\right)}(t)$ is proportional to $\dot{\lambda}_{2}(t)$. If $\lambda_{2}(t)$ changes slowly in time and $\lambda_{1}(t)$ is a fast time-varying parameter so that $A_{\alpha \gamma ; k m}^{\left(\lambda_{2}\right)}(t) \ll k \omega, \forall k$, we may ignore all the terms of $H^{F}(t)$ with $k \neq m$, i.e.,

$$
H_{\alpha, \gamma ; k, m}^{F}(t) \approx\left[A_{\alpha \gamma ; k m}^{\left(\lambda_{2}\right)}(t)-k \omega \delta_{\alpha \gamma}\right] \delta_{k m} .
$$

In analogy to the conventional adiabatic approximation, this is the adiabatic approximation of the Floquet band. Using Eq. (12), the approximate solution of Eq. (10) can be obtained as follows:

$$
U(\tau, 0)=c(\tau) \approx \mathcal{T} e^{i \int_{0}^{\tau} A_{0}^{\left(\lambda_{2}\right)}(t) d t},
$$

where $A_{0}^{\left(\lambda_{2}\right)}(t)=(1 / T) \int_{0}^{T} A^{\left(\lambda_{2}\right)}\left(\lambda_{1}, t\right) d \lambda_{1}$.

By comparing Eq. (13) with Eq. (8), it can be clearly observed that $A^{\left(\lambda_{2}\right)}\left(\lambda_{1}, t\right)$ can be replaced with an average quantity $A_{0}^{\left(\lambda_{2}\right)}(t)$, which can be interpreted as a gauge potential resulting from the adiabatic motion in a single degenerate Floquet band. However, it is still not clear what benefits this replacement will provide to quantum computing tasks and whether a universal quantum computation scheme can be constructed in practice using the gauge potential $A_{0}^{\left(\lambda_{2}\right)}(t)$. We will address these problems in the next section.

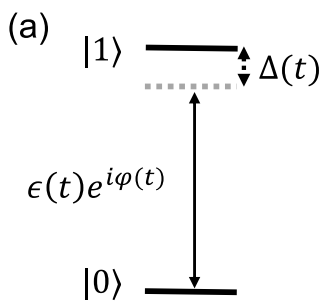

(b)

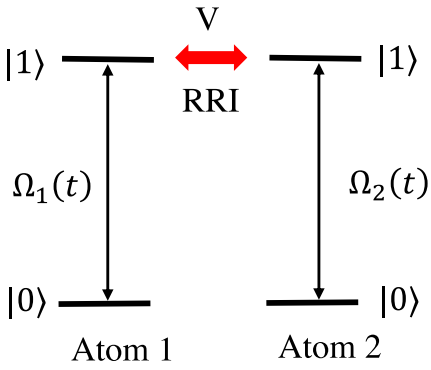

FIG. 1. (a) Setup for the two-level Rydberg atom. The two-level system is driven off-resonantly with time-dependent detuning $\Delta(t)$, Rabi frequency $\epsilon(t)$, and phase $\varphi(t)$. (b) Illustration of control of the two-qubit gate based on RRI between two identical two-level Rydberg atoms, where $V$ is the RRI strength. For individual Rydberg atoms, a resonant microwave pulse with Rabi frequency $\Omega_{\alpha}$ can be applied to facilitate the transition $|0\rangle \rightarrow|1\rangle$.

\section{FLOQUET GQC BASED ON RYDBERG ATOMS}

For universal QC, at least two types of noncommutable single-qubit gates and one nontrivial two-qubit gate are needed [87]. On the other hand, the Rydberg atom provides an appealing experimental platform for the implementation of quantum computation because of its long coherence time [88,89]. In this section, using the gauge potential $A_{0}^{\left(\lambda_{2}\right)}(t)$ in Eq. (13), we give possible physical realizations of universal single-qubit gates and a nontrivial two-qubit gate based on Rydberg atoms. They constitute a universal geometric QC scheme. This geometric QC scheme is referred to as FGQC. Notably, our theory can be applied to other platforms; only the Rydberg atom has been used to show the feasibility.

\section{A. Single-qubit gates}

Consider a Rydberg atom with the ground state and the Rydberg state encoding $|0\rangle$ and $|1\rangle$, respectively. By applying appropriately selected laser light, the ground state can be directly coupled to the Rydberg state using one photon process or via a multiphoton process using one or two intermediate (non-Rydberg) states [90]. After performing an adiabatic elimination of the intermediate state, the Hamiltonian in the rotating frame can be written as follows:

$$
H(t)=\frac{\Delta(t)}{2} \sigma_{z}+\frac{\epsilon(t)}{2}\left[\cos \varphi(t) \sigma_{x}+\sin \varphi(t) \sigma_{y}\right] .
$$

This Hamiltonian drives the transition $|0\rangle \rightarrow|1\rangle$ with Rabi frequency $\epsilon(t) \exp [i \varphi(t)]$, as shown in Fig. 1(a), where $\Delta(t)$ is the effective detuning. In an actual experiment, the laser parameters can be modulated with an acousto-optic modulator driven by an arbitrary waveform generator. By combining with some feed-forward approaches, the experimentally applied pulse can be ensured to be a faithful representation of the desired profile [88-90].

By taking $|0\rangle,|1\rangle$ as the computational basis, an arbitrary one-qubit nonadiabatic geometric gate can be achieved as follows:

$$
U(\tau, 0)=e^{i \gamma \mathbf{F} \cdot \mathbf{n}},
$$

where $\mathbf{F}=\frac{1}{2}\left(\sigma_{x}, \sigma_{y}, \sigma_{z}\right)^{\mathrm{T}}, \mathbf{n}=(\sin \theta \cos \phi, \sin \theta \sin \phi, \cos \theta)$ is an arbitrary unit vector, and $\gamma$ is an arbitrary phase with 
geometric feature. Equation (15) describes a rotational operation around the axis $\mathbf{n}$ by an angle $\gamma$. If the pulse shapes of the following form are selected,

$$
\begin{gathered}
\Delta(t)=\Delta_{0}(t) \cos \left(\omega t+\theta_{0}\right), \\
\epsilon(t)=\epsilon_{0}(t) \cos \left(\omega t+\theta_{0}\right),
\end{gathered}
$$

where $\omega$ and $\theta_{0}$ are time-independent parameters, $\Delta_{0}(t)$ and $\epsilon_{0}(t)$ are in general time-dependent real functions. Then, the Hamiltonian can be rewritten as follows:

$$
H(t)=\cos \left(\omega t+\theta_{0}\right) \mathbf{F} \cdot \mathbf{r}(t),
$$

where $\mathbf{r}(t)=\left(\epsilon_{0}(t) \cos \varphi(t), \epsilon_{0}(t) \sin \varphi(t), \Delta_{0}(t)\right)^{\mathrm{T}}$. Equation (18) has the same form as Eq. (9) if $\lambda_{1}(t)=\omega t+\theta_{0}$, $f\left[\lambda_{1}(t)\right]=\cos \left[\lambda_{1}(t)\right]$, and $H_{0}(t)=\mathbf{F} \cdot \mathbf{r}(t)$. According to Eq. (6), for a FGQC scenario, the ancillary basis is given by the transformation $R(\omega t, t)=\exp \left[-i \frac{\sin \left(\omega t+\theta_{0}\right)}{\omega} \mathbf{F} \cdot \mathbf{r}(t)\right]$. This transformation will result in the following approximated effective Hamiltonian [83]:

$$
A_{0}^{\left(\lambda_{2}\right)}(t)=\Omega(t)\left\{1-J_{0}[|\mathbf{r}(t)| / \omega]\right\} \mathbf{F} \cdot \mathbf{n}(t),
$$

where $\Omega(t) \mathbf{n}(t) \equiv \mathbf{r}(t) \times \dot{\mathbf{r}}(t) /|\mathbf{r}(t)|^{2}$ with $\mathbf{n}(t)$ a unit vector; $J_{0}(a)$ is the zeroth-order Bessel function. If the plane determined by vectors $\mathbf{r}$ and $\dot{\mathbf{r}}$ is fixed, then $\mathbf{n}(t)$ does not change, and the effective Hamiltonian (19) at different times is commutable. The time-evolution operator (13) can be simplified into $U(\tau, 0)=\exp [i \gamma(\tau) \mathbf{F} \cdot \mathbf{n}(t)]$ with $\gamma(\tau)=$ $\int_{0}^{\tau} d t \Omega(t)\left[1-J_{0}(|\mathbf{r}(t)| / \omega)\right] . U(\tau, 0)$ has the same form as (15). As $\mathbf{n}$ and $\gamma(\tau)$ can take any value, a universal singlequbit gate can be applied using the FGQC scenario.

Taking the $\mathrm{Z}$ and $\mathrm{X}$ gates as examples, we will give a possible set of pulse shapes and parameters for each gate. Moreover, to evaluate the performance of these gates with the given parameters, numerical results in the presence of both decoherence and a certain kind of systematic error will be presented (we also present similar contents for the Hadamard and $\mathrm{T}$ gates in Appendix B). To implement a single-qubit $\mathrm{Z}$ gate using FGQC, we select $\Delta_{0}(t)=0, \epsilon_{0}(t)=\Omega_{0}$, and $\varphi(t)=N t$, where $\Omega_{0}$ and $N$ are real constants which satisfy $N\left[1-J_{0}(|\mathbf{r}(t)| / \omega)\right] \tau=\pi$, and $\tau$ is the run time of the gate. Here, $|\mathbf{r}(t)|=\Omega_{0}$; the pulse shapes are shown in Fig. 2(a). In the numerical simulation, we set $\Omega_{0}=2 \times$ $2 \pi \mathrm{MHz}, \omega \approx 0.513 \times 2 \pi \mathrm{MHz}, N \approx 45.728 \times 2 \pi \mathrm{KHz}$, and the run time $\tau \approx 7.797 \mu \mathrm{s}$. For the X gate, $\epsilon_{0}(t)=\Omega_{0} \sin M t$, $\Delta_{0}(t)=\Omega_{0} \cos M t$, and $\varphi(t)=\pi / 2$ with $M=N$ real timeindependent constants. The corresponding pulse shapes are shown in Fig. 2(b). Other parameters are the same as the $\mathrm{Z}$ gate. In Figs. 2(c) and 2(d), for the $\mathrm{X}$ and $\mathrm{Z}$ gates in the absence of decoherence, the temporal evolutions of populations are shown for states $|0\rangle$ and $|1\rangle$ with a given initial state $|\psi(0)\rangle=|1\rangle$.

To evaluate the performance of FGQC in the presence of decoherence and global control error, we consider noisy time evolution which is described by the master equation as follows:

$$
\frac{d \rho(t)}{d t}=-i\left[H^{\prime}(t), \rho(t)\right]+\sum_{i=1}^{2} \mathcal{L}_{i}[\rho(t)],
$$
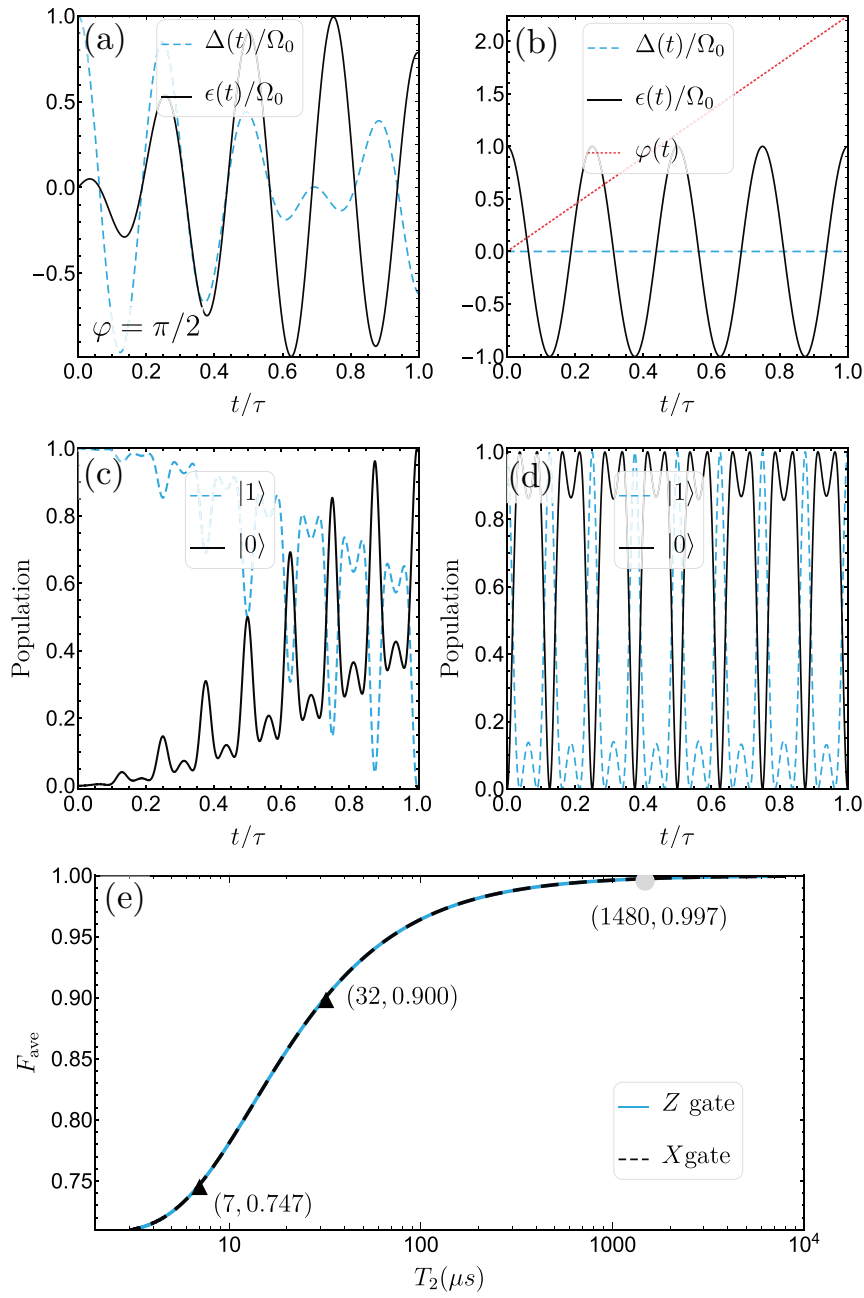

FIG. 2. (a) Detuning $\Delta(t)$ (blue dashed line) and Rabi frequency $\epsilon(t)$ (black solid line) for the numerical simulation of the $\mathrm{X}$ gate using FGQC. (b) Detuning $\Delta(t)$ (blue dashed line), Rabi frequency $\epsilon(t)$ (black solid line), and phase $\varphi(t)$ (red dotted line) for the numerical simulation of the $\mathrm{Z}$ gate using FGQC, and temporal evolution of populations (blue dashed line for state $|1\rangle$, black dot-dashed line for state $|0\rangle$ ). (e) Gate fidelities $F_{\text {ave }}$ (with $\delta=0.05$ ) vs the coherence time $T_{2}$ for the $\mathrm{X}$ (black dashed line) and $\mathrm{Z}$ (blue solid line) gates. The two solid triangles represent two landmarks in the coherence time of the Rydberg state [91,92] and its corresponding X gate fidelities; the solid gray circle represents a recent landmark in the coherence time of the superconducting fluxonium qubit [93] and its corresponding $X$ gate fidelity (rounded off to three significant figures, almost all of the $\mathrm{Z}$ gate fidelities are the same as those of the $\mathrm{X}$ gate). In the numerical simulation, we set $\gamma_{1}=\gamma_{2}$.

where $H^{\prime}(t)=(1+\delta) H(t)$ with $H(t)$ the ideal Hamiltonian, $\delta$ represents the amplitude of global control error, $\mathcal{L}_{i}[\rho]=$ $\gamma_{i}\left[A_{i} \rho A_{i}^{+}-(1 / 2)\left\{A_{i}^{+} A_{i}, \rho\right\}\right]$ is the Lindblad superoperator which acts on the density matrix $\rho$ of the quantum system, and $\{a, b\}=a b+b a$ is the anticommutator. In the case of a two-level Rydberg atom, the incoherent processes, including decay and dephasing, are $A_{1}=\sigma_{-}$and $A_{2}=\sigma_{z}$, where $\sigma_{-}$is the spin ladder operator and $\sigma_{z}$ is the Pauli $z$ matrix; thus, $\gamma_{1}$ and $\gamma_{2}$ are the decay and dephasing rates, respectively. Here, we select $\gamma_{1}=\gamma_{2}$ (i.e., $T_{1}=T_{2}$, for $T_{i}=1 / \gamma_{i}, i \in\{1,2\}$ ) and 
$\delta=0.05$. The nonunitary gate fidelity is defined by $F_{\text {ave }}=$ $(1 / 2 \pi) \int_{0}^{2 \pi} \sqrt{\left\langle\psi_{I}(\tau)|\rho(\tau)| \psi_{I}(\tau)\right\rangle} d \Theta$, for the initial states of the form $|\psi(0)\rangle=\cos \Theta|0\rangle+\sin \Theta|1\rangle$, where a total of 101 different values of $\Theta$ were uniformly selected in the range $[0,2 \pi],\left|\psi_{I}(\tau)\right\rangle$ represents the ideal final state, and $\rho(\tau)$ is the final state of the nonunitary process.

The numerical results indicate that, for the $\mathrm{X}(\mathrm{Z})$ gate, to achieve the average fidelity $F_{\text {ave }}=0.999$, the coherence time should be $T_{2} \approx 4 \mathrm{~ms}$ (4.2 ms); to achieve $F_{\text {ave }}=0.9999$, we need $T_{2} \approx 98 \mathrm{~ms}(100 \mathrm{~ms})$. The currently available coherence time of the Rydberg state is $T_{2} \approx 32 \mu \mathrm{s}$ [92], which results in $F_{X \text {,ave }} \approx 0.900\left(F_{Z \text {,ave }} \approx 0.899\right)$. Therefore, at present, the performance of FGQC based on the Rydberg state is limited by the short coherence time of the latter. We also evaluated the corresponding gate fidelities of a recent landmark coherence time of a superconducting fluxonium qubit, $T_{2} \approx 1.48 \mathrm{~ms}$ [93], and got $F_{X \text {,ave }} \approx F_{Z \text {,ave }} \approx 0.997$, with $F_{X \text {,ave }}$ and $F_{Z \text {,ave }}$ the nonunitary gate fidelities of the $X$ and $Z$ gates, respectively. In Fig. 2(e), we show how the $\mathrm{X}$ and $\mathrm{Z}$ gate fidelities vary with the coherence time, and the corresponding $X$ gate fidelities of some important coherence time (rounded off to three significant figures; almost all of the $\mathrm{Z}$ gate fidelities are the same as those of the $\mathrm{X}$ gate).

With respect to the feasibility of our proposal, a recent experimental study in Ref. [90] was selected for comparison. In this study, the amplitude of $\epsilon(t)$ can be changed from 0 to $5 \times 2 \pi \mathrm{MHz}$ within $0.5 \mu \mathrm{s}$, and the detuning $\Delta(t)$ can be changed from $-15 \times 2 \pi \mathrm{MHz}$ to a value larger than $7.5 \times$ $2 \pi \mathrm{MHz}$ within $1 \mu \mathrm{s}$. Also, the pulses in this study were given by a numerical optimization method known as RedCRAB; their shapes are not that smooth. In our proposal, taking the $\mathrm{X}$ gate as an example, the parameters $\epsilon(t)$ and $\Delta(t)$ should be changed smoothly within the range $[-2,2] \times 2 \pi \mathrm{MHz}$ in about $7.797 \mu \mathrm{s}$; clearly, the pulses in this proposal are more smooth and change more slowly within a smaller range. In other words, by comparing with recently experimental results, this proposal needs a series of smoother, more slowly varying pulses with smaller ranges. Therefore, the control in implementing the FGQC $X$ gate is achievable. The control of the FGQC $\mathrm{Z}$ gate is also slow and smooth, despite $\Delta(t)=0$; it also requires $\varphi(t)=N t$, that is, $\varphi(t)$ increasing linearly at a slow rate; to our knowledge, it is not clear weather this kind of control is achievable.

\section{B. Two-qubit gate}

It is shown that an arbitrary one-qubit FGQC gate can be obtained by addressing an individual Rydberg atom with laser pulses. To achieve universal GQC, a nontrivial two-qubit gate is needed in addition to one-qubit gates. We here demonstrate how to achieve a nontrivial two-qubit FGQC gate using the Rydberg-Rydberg interaction (RRI).

Consider two two-level Rydberg atoms with RRI $V$, as shown in Fig. 1(b). The $\alpha$ th atom is resonantly driven by a microwave pulse to achieve the transitions $|0\rangle_{\alpha} \rightarrow|1\rangle_{\alpha}$, with Rabi frequency $\Omega_{\alpha}(t)$. In the rotating frame, the Hamiltonian of the two-Rydberg-atom system can be expressed as follows [27]:

$$
H_{12}=H_{1} \otimes \mathbb{I}_{2}+\mathbb{I}_{1} \otimes H_{2}+V|11\rangle\langle 11|,
$$

where $H_{\alpha}=\Omega_{\alpha}(t)\left(|0\rangle_{\alpha}\langle 1|+| 1\rangle_{\alpha}\langle 0|\right)$ is a single-atom Hamiltonian describing the interaction between the $\alpha$ th atom and laser pulses; $\mathbb{I}_{\alpha}$ is the identity operator acting on the $\alpha$ th Rydberg atom.

To achieve a nontrivial two-qubit gate, the Rabi frequencies of laser pulses can be taken as $\Omega_{1}(t)=-\Omega_{R}(t) \cos (\phi / 2)$ and $\Omega_{2}(t)=\Omega_{R}(t) \sin (\phi / 2)$. Thus,

$$
H_{12}(t)=\Omega_{R}(t)\left(|B\rangle\left\langle 00|-| B^{\prime}\right\rangle\langle 11|+\text { H.c. }\right)+V|11\rangle\langle 11|,
$$

where $\quad|B\rangle=\sin (\phi / 2)|01\rangle-\cos (\phi / 2)|10\rangle$ and $\quad\left|B^{\prime}\right\rangle=$ $\cos (\phi / 2)|01\rangle-\sin (\phi / 2)|10\rangle$.

Further, taking a rotation $U=\exp [-i V t|11\rangle\langle 11|]$, the twoatom Hamiltonian can be recast as follows:

$$
H_{\text {rot }}(t)=\Omega_{R}(t)\left(|B\rangle\left\langle 00|-| B^{\prime}\right\rangle\langle 11| e^{-i V t}\right)+\text { H.c. }
$$

If $V \gg \Omega_{R}(t)$, the off-resonant terms are negligible, and the simultaneous excitation of two atoms from the ground state to the Rydberg states is inhibited; therefore,

$$
H_{\text {rot }}(t) \approx \Omega_{R}(t)|B\rangle\langle 00|+\text { H.c. }
$$

According to Eq. (24), the effective Hamiltonian only affects subspace $S=\operatorname{Span}\{|B\rangle,|00\rangle\}$. Therefore, $S$ was set as the computational space. Consider $\Omega_{R}(t)=$ $\left[\Omega_{0} / 2\right] f(\omega t) \exp [i \varphi(t)]$ where $\Omega_{0}$ is a real constant. Both $f(\omega t)$ and $\varphi(t)$ are real functions. The Hamiltonian (24) can be rewritten as follows:

$$
H_{\mathrm{rot}}(\omega t, t)=f(\omega t) \mathbf{F} \cdot \mathbf{r}^{\prime}(t)
$$

where $\quad \mathbf{F} \equiv\left(\tilde{\sigma}_{x}, \tilde{\sigma}_{y}, \tilde{\sigma}_{z}\right)^{T} / 2 \quad$ and $\quad \mathbf{r}^{\prime}(t)=\Omega_{0}(\cos \varphi(t)$, $-\sin \varphi(t), 0)^{T} \quad$ with $\quad \tilde{\sigma}_{x} \equiv|B\rangle\langle 00|+| 00\rangle\langle B|, \quad \tilde{\sigma}_{y} \equiv$ $-i|B\rangle\langle 00|+i| 00\rangle\langle B|, \quad$ and $\quad \tilde{\sigma}_{z} \equiv|B\rangle\langle B|-| 00\rangle\langle 00|$. To be more specific, we considered $f(\omega t)=\cos \omega t$; then, $H_{\text {rot }}(\omega t, t)=\cos \omega t \mathbf{F} \cdot \mathbf{r}^{\prime}(t)$. To construct a FGQC gate, select $R(t)=\exp \left[-i \Omega_{0} \sin \omega t \mathbf{F} \cdot \mathbf{r}^{\prime}(t) / \omega\right]$, satisfying the boundary condition $R(\tau)=R(0)=I$ if $\omega \tau=k \pi, \forall k \in N^{+}$. The corresponding effective Hamiltonian can be written as follows:

$$
H_{\mathrm{eff}}(t)=\dot{\varphi}(t) R^{\dagger}(t)(-i \partial / \partial \varphi) R(t)
$$

Similar to the derivation of Eq. (13), if $\omega \gg \dot{\varphi}(t)$, then at $t=$ $\tau$ the time-evolution operator can be approximated as follows:

$$
U(\tau, 0) \approx \mathcal{T} e^{-i \int_{0}^{\tau} H_{\mathrm{eff}}^{(0)}(t) d t},
$$

where $H_{\mathrm{eff}}^{(0)}(t)=\left[1-J_{0}\left(\Omega_{0} / \omega\right)\right] \mathbf{F} \cdot\left(\mathbf{r}^{\prime} \times \dot{\mathbf{r}}^{\prime}\right) / \Omega_{0}^{2}$. If $\varphi(t)=$ $N t$, then $\mathbf{r}^{\prime} \times \dot{\mathbf{r}}^{\prime} / \Omega_{0}^{2}=-N(0,0,1)^{T}$ and $H_{\mathrm{eff}}^{(0)}(t)=-N[1-$ $\left.J_{0}\left(\Omega_{0} / \omega\right)\right] \tilde{\sigma}_{z} / 2$.

In terms of the basis $\{|00\rangle,|01\rangle,|10\rangle,|11\rangle\}$, the matrix form of the average effective Hamiltonian can be expressed as follows:

$$
H_{\mathrm{eff}}^{(0)}(t)=C \cdot\left(\begin{array}{cccc}
0 & 0 & 0 & 0 \\
0 & \cos ^{2}\left(\frac{\phi}{2}\right) & \frac{-\sin \phi}{2} & 0 \\
0 & \frac{-\sin \phi}{2} & \sin ^{2}\left(\frac{\phi}{2}\right) & 0 \\
0 & 0 & 0 & -1
\end{array}\right),
$$


where $C \equiv-(N / 2)\left[1-J_{0}\left(\Omega_{0} / \omega\right)\right]$. The time-evolution operator is given by

$$
\begin{aligned}
& U(\tau, 0) \\
& =\left(\begin{array}{cccc}
1 & 0 & 0 & 0 \\
0 & \frac{1}{2}\left(D_{1}-D_{2} \cos \phi\right) & \frac{1}{2} D_{2} \sin \phi & 0 \\
0 & \frac{1}{2} D_{2} \sin \phi & \frac{1}{2}\left(D_{1}+D_{2} \cos \phi\right) & 0 \\
0 & 0 & 0 & e^{i C \tau}
\end{array}\right),
\end{aligned}
$$

where $D_{1}=1+\exp (-i C \tau)$ and $D_{2}=1-\exp (-i C \tau)$. Clearly, when $e^{i C \tau}=-1$ and $\phi=\pi / 2$, we have

$$
U(\tau, 0)=\left(\begin{array}{cccc}
1 & 0 & 0 & 0 \\
0 & 0 & 1 & 0 \\
0 & 1 & 0 & 0 \\
0 & 0 & 0 & -1
\end{array}\right) .
$$

This operation is a SWAP-like gate because

$$
\begin{aligned}
& U(\tau, 0)|10\rangle=|01\rangle, \\
& U(\tau, 0)|01\rangle=|10\rangle,
\end{aligned}
$$

which is a nontrivial two-qubit gate ensuring that universal quantum computation can be applied using FGQC.

\section{The robustness of FGQC against global control error}

Since only a gauge potential $A_{0}^{\left(\lambda_{2}\right)}(t)$ can be attributed to the cyclic unitary evolution operator (13), FGQC is a geometric scenario. However, as shown in Ref. [44], a geometric scenario is not necessarily resistant to global control error. Here, we will demonstrate that the FGQC is a resistant GQC scenario to global control error. To prove this, we consider a quantum system which is subject to global control error. Given the initial state $\left|\psi_{m}(0)\right\rangle$, the corresponding time-evolution state can be expressed as $\left|\psi_{m}^{\prime}(\tau)\right\rangle=$ $U^{\prime}(\tau, 0)\left|\psi_{m}(0)\right\rangle$ with $U^{\prime}(\tau, 0)=\mathcal{T} \exp \left\{i \int_{0}^{\tau} d t\left[A^{\left(\lambda_{2}\right)}(\omega t, t)-\right.\right.$ $\delta H(\omega t, t)]\}$. Normally, $\delta$ is a small quantity in experiment and it is reasonable to assume that $\delta H_{\alpha \gamma}(t) \ll k \omega$. Similar to the derivation of Eq. $(13), U^{\prime}(\tau, 0)$ can be approximately expressed as follows:

$$
U^{\prime}(\tau, 0) \approx \mathcal{T} e^{i \int_{0}^{\tau}\left[A_{0}^{\left(\lambda_{2}\right)}(t)-\delta \bar{H}(t)\right] d t},
$$

where $\bar{H}(t)=(1 / T) \int_{0}^{2 \pi} H\left(\lambda_{1}, t\right) d \lambda_{1}$; then, using perturbation theory up to $\mathcal{O}(\delta)$,

$$
\left|\psi_{m}^{\prime}(\tau)\right\rangle=\left|\psi_{m}(\tau)\right\rangle-\delta \cdot i \sum_{n}\left|\psi_{n}(\tau)\right\rangle Q_{n m}(\tau)+\mathcal{O}\left(\delta^{2}\right),
$$

where $\left|\psi_{m}(\tau)\right\rangle=U(\tau, 0)\left|\psi_{m}(0)\right\rangle$ is the ideal time-evolution state and $Q_{n m}(t)=\int_{0}^{t} d t^{\prime}\left\langle\psi_{n}\left(t^{\prime}\right)\left|\bar{H}\left(t^{\prime}\right)\right| \psi_{m}\left(t^{\prime}\right)\right\rangle$.

Equation (34) clearly shows that to eliminate the second term at the right side $Q_{m n}(\tau)$ should be equal to zero:

$$
\int_{0}^{\tau}\left\langle\psi_{n}(t)|\bar{H}(t)| \psi_{m}(t)\right\rangle d t=0 \quad \forall m, n .
$$

Equation (35) maintains the robustness of FGQC against global control error to the first order in $\delta$. In a FGQC scenario,
$\bar{H}$ is given by

$$
\bar{H}(t)=\bar{f}\left(\lambda_{1}\right) H_{0}\left(\lambda_{2}\right),
$$

where $\bar{f}\left(\lambda_{1}\right)=(1 / T) \int_{0}^{T} f\left(\lambda_{1}\right) d \lambda_{1}$ and $f\left(\lambda_{1}\right)$ is a periodic function with period $T$. In this paper, $f\left(\lambda_{1}\right)=\cos \left(\lambda_{1}\right), \bar{f}\left(\lambda_{1}\right)$ is equal to zero, $\bar{H}(t)=0$, and Eq. (35) is satisfied. The above analysis is based on single-qubit FGQC theory, but it can also be applied to the two-qubit gate case. For the two-qubit case, one only need replace $A_{0}^{\left(\lambda_{2}\right)}(t)$ and $H(t)$ with $H_{\text {eff }}^{(0)}(t)$ and $H_{\mathrm{rot}}(t)$, respectively. Therefore, FGQC is resistant to global control error.

\section{Numerical evidence of the robustness of FGQC against global control error}

In Figs. 3 and 4, numerical results of the single-qubit $X$ and $\mathrm{Z}$ gates and the two-qubit gate described in Eq. (30) are given. They show the single- and two-qubit gate fidelities vs $\delta$, respectively; clearly, the FGQC scenario is more robust than the standard dynamical scenario and the NGQC scenario in solving global control errors (see Appendix A for details of the standard dynamical and NGQC gates). The parameters of the FGQC two-qubit gate were selected as follows: $\Omega_{0}=2 \times 2 \pi \mathrm{MHz}, \omega \approx 0.52 \times 2 \pi \mathrm{MHz}, N \approx 0.58 \mathrm{MHz}$, $V \approx 0.33 \mathrm{GHz}$, and the run time $\tau \approx 7.7 \mu \mathrm{s}$. Due to the presence of the $\left|B^{\prime}\right\rangle\langle 11|$ term and its hermitian conjugation, the fidelity at $\delta=0$ is not 1 : for FGQC and NGQC, $F(\delta=$ $0) \approx 0.9982$ and 0.9989 , respectively. The single-qubit FGQC $\mathrm{T}$ and Hadamard gates were also simulated, and the results are shown in Appendix B.

For a time-dependent error $\delta=\delta(t)$, we should modify the unitary time-evolution operator in Eq. (33) by replacing $\delta \bar{H}(t)$

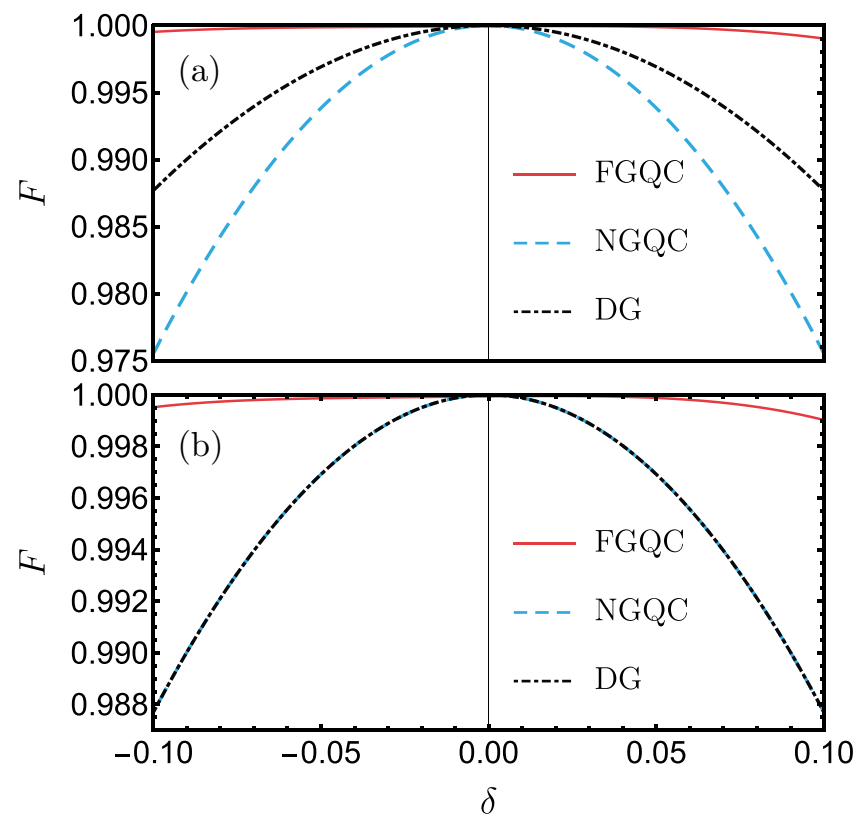

FIG. 3. Fidelities of the $\mathrm{Z}$ (a) and $\mathrm{X}$ (b) gates vs the amplitude of the global control error (without considering decoherence in the simulation). The results of three different protocols are shown: FGQC (red solid line), NGQC (blue dashed line), and standard DG (black dot-dashed line). 


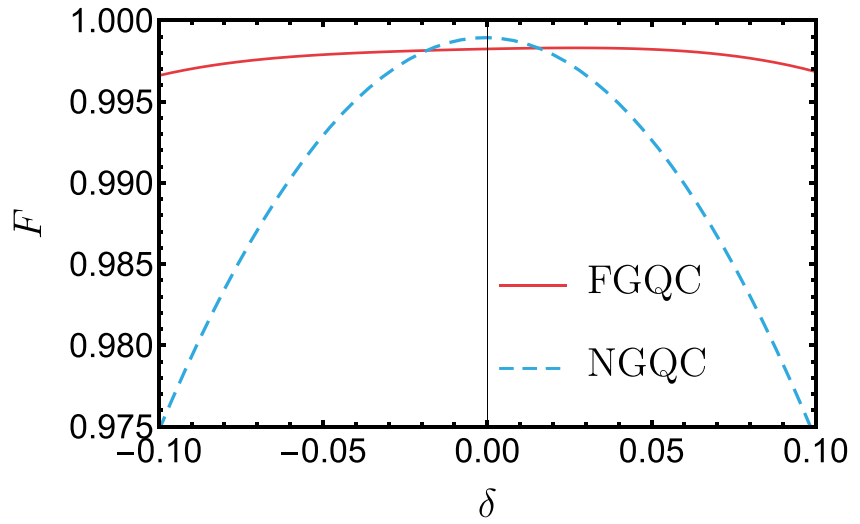

FIG. 4. The fidelity $F$ vs the amplitude $\delta$ of global control error for the same two-qubit gate using FGQC (red solid line) and NGQC (blue dashed line), respectively. The two-qubit gate is given by Eq. (30).

in the exponent with the following expression:

$$
(1 / T) \int_{0}^{2 \pi} \delta(t) H\left(\lambda_{1}, t\right) d \lambda_{1} .
$$

In our proposal, $\lambda_{1}=\omega t$ and $H\left(\lambda_{1}, t\right)=\cos (\omega t) H_{0}(t)$ with $\omega$ a time-independent real number and $H_{0}(t)$ a slowly varying operator; the expression Eq. (37) can be rewritten as $(1 / T) \int_{0}^{2 \pi} d t \cos (\omega t) \delta(t) H_{0}(t)$. Clearly, when $\delta(t)$ is slow enough, this expression is approximately equal to zero. Therefore, FGQC is still a robust scenario when $\delta(t)$ is slow enough. A numerical demonstration is also given through simulating a $\mathrm{Z}$ gate in the presence of $\delta(t)$. A stochastic process called the Ornstein-Uhlenbeck (O-U) process is considered to describe the time dependence of $\delta(t)$. To be specific, the O-U process is a stochastic process with power spectral density $S(\omega)=$ $\frac{\Gamma}{\pi\left(\Gamma^{2}+\omega^{2}\right)}$, where $1 / \Gamma$ is the correlation time. The Hamiltonian is given by

$$
H^{\prime}(t)=[1+M \delta(t)] H(t),
$$

where $H(t)$ is the ideal Hamiltonian and $M$ is a zoom factor. In the simulation, we set $\Gamma \tau=0.001$. For each $M$, we first sampled 100 such processes randomly, $\left\{\delta_{i}(t) \mid i=1, \ldots, 100\right\}$; then, we calculate the gate fidelity for each $\delta_{i}(t)$ and obtain the average gate fidelity for these 100 processes. The results are shown in Fig. 5.

\section{CONCLUSION}

A geometric scheme called FGQC is reported. In a FGQC scenario, error-resilient geometric gates based on periodically driven two-level systems can be constructed via a non-Abelian geometric phase. This non-Abelian geometric phase emerges from a periodically driven quantum system and was found in a recent study [83]. To construct FGQC, possible implementations of universal single-qubit gates and a nontrivial two-qubit gate using Rydberg atoms were proposed. To numerically evaluate its realistic performance, the $\mathrm{X}$ and $\mathrm{Z}$ gates were simulated in the presence of both decoherence and global control error using recent experimental parameters. The numerical results indicate that, at present, the performance of FGQC

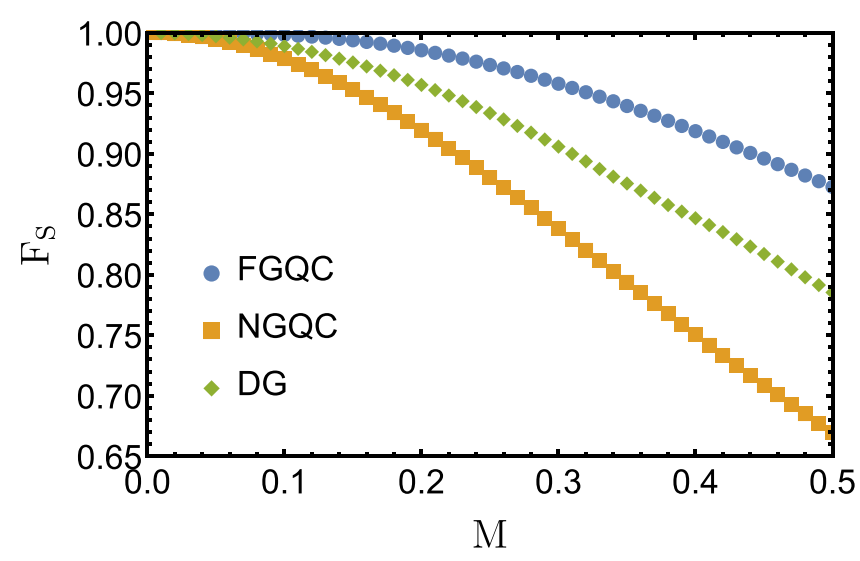

FIG. 5. The average gate fidelity $F_{S}$ vs $M$ for the $\mathrm{Z}$ gate based on FGQC (blue dots), NGQC (yellow rectangle), and DG (green diamond), respectively.

based on the Rydberg state is limited by the short coherence time of the latter: for the currently available coherence time of the Rydberg state $\left(T_{2} \approx 32 \mu\right.$ s [92]), we got $F_{X \text {,ave }} \approx 0.900$ $\left(F_{Z \text {,ave }} \approx 0.899\right)$. Moreover, the robustness of FGQC against global control error was analytically demonstrated using perturbation method. By comparing the numerical results of FGQC with NGQC and DG, the superiority of FGQC in solving global control error was confirmed. Because FGQC is based on two-level systems, compared with NHQC, it has the advantage of not requiring complex quantum control on a multilevel structure. Although the FGQC scenario is sensitive to decoherence, as quantum technologies evolve, the coherence time of various qubits (e.g., qubits based on the Rydberg state [92] and superconducting qubits [93]) grows; in the future, FGQC may become a competitive quantum gate architecture approach.

\section{ACKNOWLEDGMENTS}

This work was supported by the Natural Science Foundation of Guangdong Province (Grant No. 2017B030308003); the Key-Area Research and Development Program of Guangdong Province (Grant No. 2018B030326001); the Science, Technology, and Innovation Commission of Shenzhen Municipality (Grants No. JCYJ20170412152620376，No. JCYJ20170817105046702, and No. KYTDPT20181011104202253); the National Natural Science Foundation of China (Grants No. 11875160, No. U1801661, and No. 11804308); the Economy, Trade, and Information Commission of Shenzhen Municipality (Grant No. 201901161512); and the Guangdong Provincial Key Laboratory (Grant No. 2019B121203002).

\section{APPENDIX A: DETAILS OF OTHER SCENARIOS}

\section{Simulating the $Z$ gate: The NGQC $Z$ gate}

The corresponding Hamiltonian reads

$$
H(t)=\left(\Omega_{0} / 2\right) e^{-i \phi(t)}|1\rangle\langle 0|+\text { H.c. },
$$


where

$$
\phi(t)= \begin{cases}\pi & 0 \leqslant t<\tau_{\mathrm{N} 1 \mathrm{Z}}, \\ -\pi / 2 & \tau_{\mathrm{N} 1 \mathrm{Z}} \leqslant t \leqslant \tau_{\mathrm{NZ}},\end{cases}
$$

with $\tau_{\mathrm{N} 1 \mathrm{Z}}=\tau_{\mathrm{NZ}} / 2$ and the run time $\tau_{\mathrm{NZ}}=2 \pi / \Omega_{0}=0.5 \mu \mathrm{s}$.

\section{Simulating the $Z$ gate: The standard dynamical $Z$ gate}

For this scenario, the Hamiltonian can be written in the same form as Eq. (14) in the main text, but with $\Delta(t)=\Omega_{0}$, $\epsilon(t)=0$, and the run time $\tau_{\mathrm{DSZ}}=\pi / \Omega_{0}=0.25 \mu \mathrm{s}$.

\section{Simulating the $X$ gate: The NGQC $X$ gate}

The Hamiltonian of this scenario can be written in the same form as Eq. (A1) but with

$$
\phi(t)= \begin{cases}-\pi / 2 & 0 \leqslant t<\tau_{\mathrm{NX} 1}, \\ \pi & \tau_{\mathrm{NX} 1} \leqslant t<\tau_{\mathrm{NX} 2}, \\ -\pi / 2 & \tau_{\mathrm{NX} 2} \leqslant t \leqslant \tau_{\mathrm{NX}},\end{cases}
$$

where $\quad \tau_{\mathrm{NX} 1}=(1 / 4) \tau_{\mathrm{NX}}, \quad \tau_{\mathrm{NX} 2}=(3 / 4) \tau_{\mathrm{NX}}, \quad$ and $\quad \tau_{\mathrm{NX}}=$ $2 \pi / \Omega_{0}=0.5 \mu \mathrm{s}$.

\section{Simulating the $X$ gate: The standard dynamical $X$ gate}

The Hamiltonian of this scenario can be written in the same form as Eq. (14) in the main text with $\Delta(t)=0$ and $\varphi(t)=0$; we set $\epsilon(t)=\Omega_{0}$ and the run time $\tau_{\mathrm{DSX}}=\pi / \Omega_{0}=0.25 \mu \mathrm{s}$.

\section{Details of the NGQC two-qubit gate}

The corresponding Hamiltonian of the NGQC two-qubit gate [27] can be written in the form of Eq. (23) in the main text, but $\Omega_{R}(t)$ is time independent. We set $\Omega_{R}(t)=\Omega_{0}$, the run time $\tau=2 \pi / \Omega_{0}=0.5 \mu \mathrm{s}$, and other parameters are the same as the FGQC gate.

\section{APPENDIX B: TWO MORE GATES: THE T AND HADAMARD GATES}

To illustrate the universality of the FGQC, we numerically simulated the $\mathrm{T}$ and Hadamard gates using FGQC theory. For both the $\mathrm{T}$ and Hadamard gates, the Hamiltonian can be written in the following form:

$$
H(t)=\frac{\Delta(t)}{2} \sigma_{z}+\frac{\epsilon_{x}(t)}{2} \sigma_{x}+\frac{\epsilon_{y}(t)}{2} \sigma_{y} .
$$

For the FGQC Hadamard gate, $\Delta(t)=-\Omega_{0} \cos (\omega t)$ $\sin \left(N_{H} t\right) / \sqrt{2}, \quad \epsilon_{x}(t)=\Omega_{0} \cos (\omega t) \sin \left(N_{H} t\right) / \sqrt{2}, \quad$ and $\epsilon_{y}(t)=\Omega_{0} \cos (\omega t) \cos \left(N_{H} t\right)$ with $\Omega_{0}=2.0 \times 2 \pi \quad \mathrm{MHz}$, $\omega \approx 0.513 \times 2 \pi \quad \mathrm{MHz}, \quad N_{H} \approx 45.728 \times 2 \pi \mathrm{KHz}, \quad$ and the run time $\tau_{H} \approx 7.797 \mu \mathrm{s}$. This kind of Hamiltonian can be obtained through applying a rotating frame transformation $\quad H_{0}(t) \rightarrow H(t)=U_{0}^{\dagger}(t) H_{0}(t) U_{0}(t) \quad$ to $H_{0}(t)=\cos (\omega t)\left[\sin \left(N_{H} t\right) \sigma_{x}+\cos \left(N_{H} t\right) \sigma_{y}\right]$ with $U_{0}=$ $\exp \left[-i(\pi / 4) \sigma_{y} / 2\right]$. For the FGQC T gate $\Delta(t)=0$, $\epsilon_{x}(t)=\Omega_{0} \cos (\omega t) \cos \left(N_{T} t\right), \quad \epsilon_{y}(t)=\Omega_{0} \cos (\omega t) \sin \left(N_{T} t\right)$ with $\Omega_{0}=2.0 \times 2 \pi \quad \mathrm{MHz}, \quad \omega \approx 0.513 \times 2 \pi \quad \mathrm{MHz}$, $N_{T} \approx 45.728 \times 2 \pi \mathrm{KHz}$, and the run time $\tau=1.95 \mu \mathrm{s}$. The Hamiltonian of the FGQC $\mathrm{T}$ and $\mathrm{Z}$ gate has the same form, and the parameters of these two gates are almost the same except the run time of the $\mathrm{T}$ gate is shorter. The
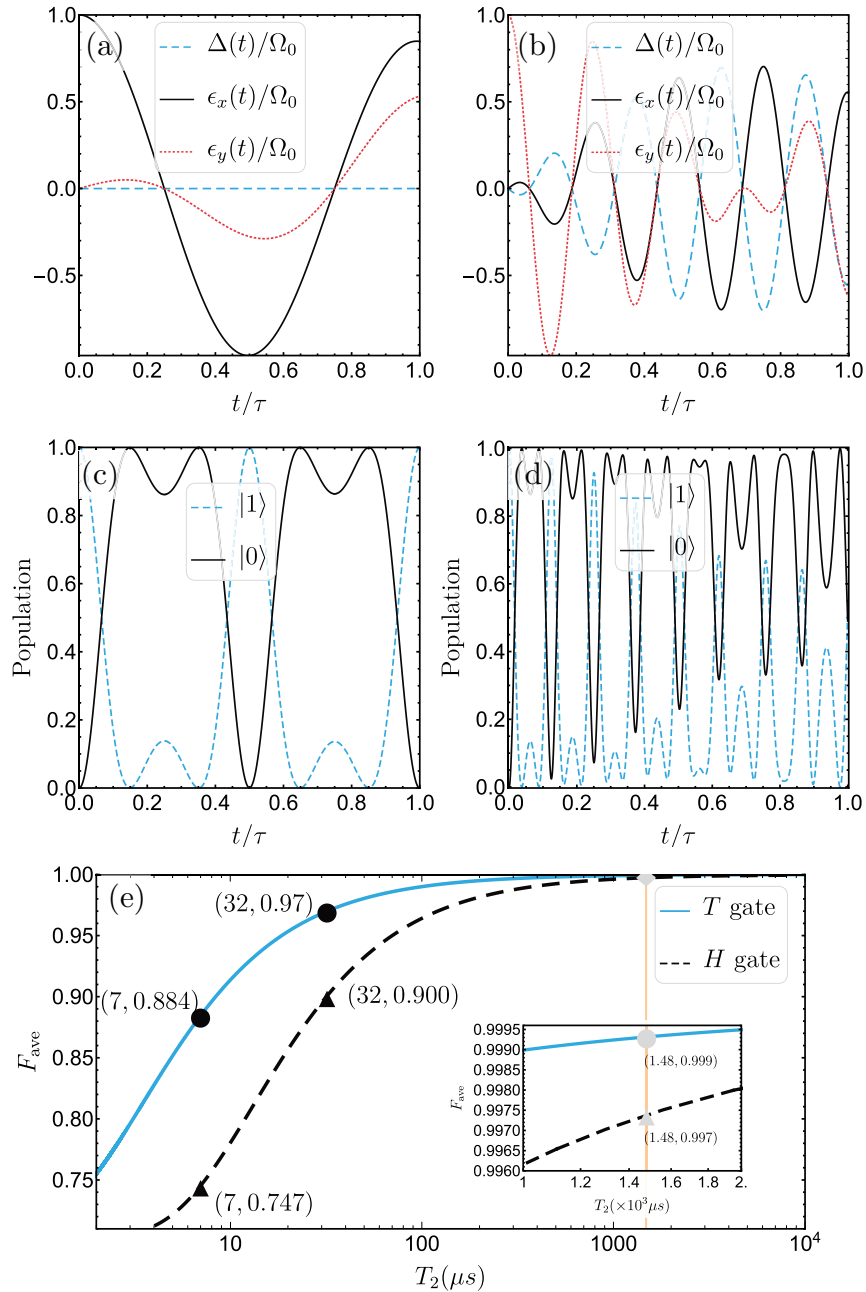

FIG. 6. The pulse shapes in numerically simulating the $\mathrm{T}$ (a) and Hadamard (b) gates. The temporal evolutions of populations with a given initial state $|\psi(0)\rangle=|1\rangle$ for the FGQC T (c) and Hadamard (d) gates are shown. (e) Gate fidelities $F_{\text {ave }}$ (with $\delta=0.05$ ) vs the coherence time $T_{2}$ for the $\mathrm{T}$ (blue solid line) and Hadamard (black dashed line) gates. The solid circles (triangles) represent gate fidelities of two landmark coherence times of the Rydberg state [91,92] for the $\mathrm{T}$ (Hadamard) gate. In the inset of (e), the solid gray circle (triangle) represents the $\mathrm{T}$ (Hadamard) corresponding gate fidelity of a recent landmark coherence time of the superconducting fluxonium qubit [93]. In the numerical simulation, we set $\gamma_{1}=\gamma_{2}$.

pulse shapes of the Hadamard and $\mathrm{T}$ gates are shown in Figs. 6(a) and 6(b), respectively. In Figs. 6(c) and 6(d), for the Hadamard and the T gate, respectively, we show the temporal evolution of state populations for $|0\rangle$ and $|1\rangle$ with a given initial state $|\psi(0)\rangle=|1\rangle$.

To evaluate the realistic performance of the FGQC $\mathrm{T}$ and $\mathrm{H}$ gates, we have numerically investigated their gate fidelities in the presence of both global control error and decoherence. The time evolution is described by the master equation in Eq. (20). In the simulation, we set $\delta=0.05$ and $\gamma_{1}=\gamma_{2}$ (i.e., $T_{1}=T_{2}$ ). The numerical results indicate that for the Rydberg state's currently available coherence time $\left(T_{2} \approx 32 \mu\right.$ s [92]), we got the T gate fidelity $F_{T \text {,ave }} \approx 0.97$ and the Hadamard gate fidelity $F_{H \text {,ave }} \approx 0.90$. We also evaluated the corresponding 

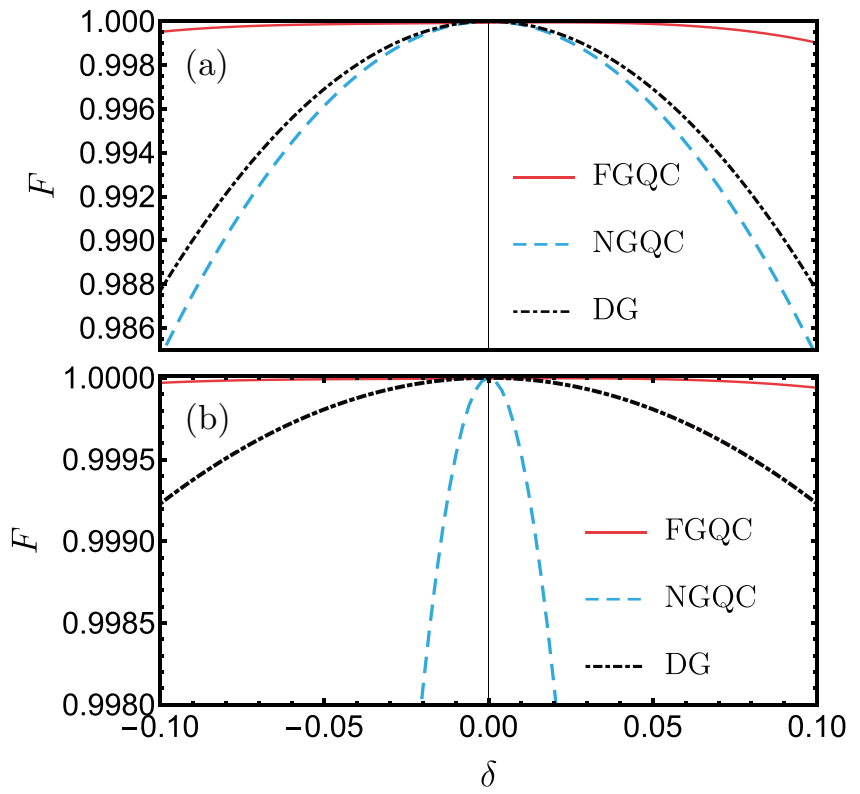

FIG. 7. Fidelities of the Hadamard (a) and T (b) gates vs the amplitude of the global control error (without considering the decoherence in the simulation). Results of four different protocols are shown: FGQC (red solid line), NGQC (blue dashed line), and standard DG (black dot-dashed line).

gate fidelities of a recent landmark coherence time of the superconducting fluxonium qubit [93], $T_{2} \approx 1.48 \mathrm{~ms}$, and got $F_{T, \text { ave }} \approx 0.999, F_{H \text {,ave }} \approx 0.997$. The relative results are shown in Fig. 6(e). In terms of the robustness against the global control error, we compare the performance of the FGQC Hadamard and $\mathrm{T}$ gates with that of other typical scenarios based on two-level systems: the NGQC and the standard DG gates. The results are shown in Fig. 7. It is readily seen from these figures that the FGQC scenarios perform much better than the other two scenarios.

\section{Simulating the $T$ gate: The NGQC $T$ gate}

We set $\Omega_{0}=2 \times 2 \pi \mathrm{MHz}$ hereinafter. The Hamiltonian of this scenario can be written as

$$
H_{\mathrm{NT}}=\left(\Omega_{0} / 2\right) e^{-i \phi(t)}|1\rangle\langle 0|+\text { H.c. }
$$

where

$$
\phi(t)= \begin{cases}\pi / 2 & 0 \leqslant t<\tau_{\mathrm{NT}} / 2, \\ \pi / 2+\pi / 8 & \tau_{\mathrm{NT}} / 2 \leqslant t \leqslant \tau_{\mathrm{NT}}\end{cases}
$$

and the run time $\tau_{\mathrm{NT}}=2 \pi / \Omega_{0}=0.5 \mu \mathrm{s}$.

\section{Simulating the $\mathbf{T}$ gate: The standard dynamical $\mathbf{T}$ gate}

The corresponding Hamiltonian reads $H(t)=\Omega_{0} \sigma_{z} / 2$ and the run time is given by $\tau_{\mathrm{DGT}}=(\pi / 4) / \Omega_{0}=0.0625 \mu \mathrm{s}$.

\section{Simulating the Hadamard gate: The NGQC Hadamard gate}

The Hamiltonian has the same form as Eq. (B2), but the time-dependent phase $\phi(t)$ is given by the following expression:

$$
\phi(t)= \begin{cases}-\pi / 2 & 0 \leqslant t<\tau_{\mathrm{NH} 1}, \\ 0 & \tau_{\mathrm{NH} 1} \leqslant t<\tau_{\mathrm{NH} 2}, \\ -\pi / 2 & \tau_{\mathrm{NH} 2} \leqslant t \leqslant \tau_{\mathrm{NH}},\end{cases}
$$

with $\tau_{\mathrm{NH} 1}=\tau_{\mathrm{NH}} / 8, \quad \tau_{\mathrm{NH} 2}=5 \tau_{\mathrm{NH}} / 8$, and $\tau_{\mathrm{NH}}=2 \pi / \Omega_{0}=$ $0.5 \mu \mathrm{s}$.

\section{Simulating the Hadamard gate: The standard dynamical Hadamard gate}

The Hamiltonian reads $H(t)=\Omega_{0}\left(\sigma_{x}+\sigma_{z}\right) / 2 \sqrt{2}$, and the run time $\tau_{\mathrm{DGH}}=\pi / \Omega_{0}=0.25 \mu \mathrm{s}$.
[1] R. P. Feynman, Simulating physics with computers, Int. J. Theor. Phys. 21, 467 (1982).

[2] Z. Li, H. Zhou, C. Ju, H. Chen, W. Zheng, D. Lu, X. Rong, C. Duan, X. Peng, and J. Du, Experimental Realization of a Compressed Quantum Simulation of a 32-spin Ising Chain, Phys. Rev. Lett. 112, 220501 (2014).

[3] L. M. K. Vandersypen, M. Steffen, G. Breyta, C. S. Yannoni, M. H. Sherwood, and I. L. Chuang, Experimental realization of Shor's quantum factoring algorithm using nuclear magnetic resonance, Nature (London) 414, 883 (2001).

[4] N. Xu, J. Zhu, D. Lu, X. Zhou, X. Peng, and J. Du, Quantum Factorization of 143 on a Dipolar-Coupling Nuclear Magnetic Resonance System, Phys. Rev. Lett. 108, 130501 (2012).

[5] E. Martín-López, A. Laing, T. Lawson, R. Alvarez, X.-Q. Zhou, and J. L. O'Brien, Experimental realization of Shor's quantum factoring algorithm using qubit recycling, Nat. Photonics 6, 773 (2012).

[6] L. K. Grover, Quantum Mechanics Helps in Searching for A Needle in a Haystack, Phys. Rev. Lett. 79, 325 (1997).
[7] P. Rebentrost, M. Mohseni, and S. Lloyd, Quantum Support Vector Machine for Big Data Classification, Phys. Rev. Lett. 113, 130503 (2014).

[8] Z. Li, X. Liu, N. Xu, and J. Du, Experimental Realization of a Quantum Support Vector Machine, Phys. Rev. Lett. 114, 140504 (2015).

[9] I. Cong, S. Choi, and M. D. Lukin, Quantum convolutional neural networks, Nat. Phys. 15, 1273 (2019).

[10] M. V. Berry, Quantal phase factors accompanying adiabatic changes, Proc. R. Soc. A 392, 45 (1984).

[11] Y. Aharonov and J. Anandan, Phase Change During a Cyclic Quantum Evolution, Phys. Rev. Lett. 58, 1593 (1987).

[12] F. Wilczek and A. Zee, Appearance of Gauge Structure in Simple Dynamical Systems, Phys. Rev. Lett. 52, 2111 (1984).

[13] J. Anandan, Non-adiabatic non-Abelian geometric phase, Phys. Lett. A 133, 171 (1988).

[14] G. D. Chiara and G. M. Palma, Berry Phase for a Spin $1 / 2$ particle in a Classical Fluctuating Field, Phys. Rev. Lett. 91, 090404 (2003). 
[15] S.-L. Zhu and P. Zanardi, Geometric quantum gates that are robust against stochastic control errors, Phys. Rev. A 72, 020301 (2005).

[16] P. J. Leek, J. M. Fink, A. Blais, R. Bianchetti, M. Göppl, J. M. Gambetta, D. I. Schuster, L. Frunzio, R. J. Schoelkopf, and A. Wallraff, Observation of Berry's phase in a solid-state qubit, Science 318, 1889 (2007).

[17] S. Filipp, J. Klepp, Y. Hasegawa, C. Plonka-Spehr, U. Schmidt, P. Geltenbort, and H. Rauch, Experimental Demonstration of the Stability of Berry's Phase for a Spin-1/2 Particle, Phys. Rev. Lett. 102, 030404 (2009).

[18] S. Berger, M. Pechal, A. A. Abdumalikov, Jr., C. Eichler, L. Steffen, A. Fedorov, A. Wallraff, and S. Filipp, Exploring the effect of noise on the Berry phase, Phys. Rev. A 87, 060303(R) (2013).

[19] J. A. Jones, V. Vedral, A. Ekert, and G. Castagnoli, Geometric quantum computation using nuclear magnetic resonance, Nature (London) 403, 869 (2000).

[20] L.-M. Duan, J. I. Cirac, and P. Zoller, Geometric manipulation of trapped ions for quantum computation, Science 292, 1695 (2001).

[21] L.-A. Wu, P. Zanardi, and D. A. Lidar, Holonomic Quantum Computation in Decoherence-Free Subspaces, Phys. Rev. Lett. 95, 130501 (2005).

[22] H. Wu, E. M. Gauger, R. E. George, M. Möttönen, H. Riemann, N. V. Abrosimov, P. Becker, H.-J. Pohl, K. M. Itoh, M. L. W. Thewalt, and J. J. L. Morton, Geometric phase gates with adiabatic control in electron spin resonance, Phys. Rev. A 87, 032326 (2013).

[23] Y.-Y. Huang, Y.-K. Wu, F. Wang, P.-Y. Hou, W.-B. Wang, W.-G. Zhang, W.-Q. Lian, Y.-Q. Liu, H.-Y. Wang, H.-Y. Zhang, L. He, X.-Y. Chang, Y. Xu, and L.-M. Duan, Experimental Realization of Robust Geometric Quantum Gates with Solid-State Spins, Phys. Rev. Lett. 122, 010503 (2019).

[24] X.-B. Wang and M. Keiji, Nonadiabatic Conditional Geometric Phase Shift with NMR, Phys. Rev. Lett. 87, 097901 (2001).

[25] S.-L. Zhu and Z. D. Wang, Implementation of Universal Quantum Gates Based on Nonadiabatic Geometric Phases, Phys. Rev. Lett. 89, 097902 (2002).

[26] J. T. Thomas, M. Lababidi, and M. Tian, Robustness of singlequbit geometric gate against systematic error, Phys. Rev. A 84, 042335 (2011).

[27] P. Z. Zhao, X.-D. Cui, G. F. Xu, E. Sjöqvist, and D. M. Tong, Rydberg-atom-based scheme of nonadiabatic geometric quantum computation, Phys. Rev. A 96, 052316 (2017).

[28] K. Z. Li, P. Z. Zhao, and D. M. Tong, Approach to realizing nonadiabatic geometric gates with prescribed evolution paths, Phys. Rev. Res. 2, 023295 (2020).

[29] T. Chen and Z.-Y. Xue, Nonadiabatic Geometric Quantum Computation with Parametrically Tunable Coupling, Phys. Rev. Appl. 10, 054051 (2018).

[30] C. Zhang, T. Chen, S. Li, X. Wang, and Z.-Y. Xue, High-fidelity geometric gate for silicon-based spin qubits, Phys. Rev. A 101, 052302 (2020).

[31] B.-J. Liu, X.-K. Song, Z.-Y. Xue, X. Wang, and M.-H. Yung, Plug-and-Play Approach to Nonadiabatic Geometric Quantum Gates, Phys. Rev. Lett. 123, 100501 (2019).

[32] E. Sjöqvist, D. M. Tong, L. Mauritz Andersson, B. Hessmo, M. Johansson, and K. Singh, Non-adiabatic holonomic quantum computation, New J. Phys. 14, 103035 (2012).
[33] G. F. Xu, J. Zhang, D. M. Tong, E. Sjöqvist, and L. C. Kwek, Nonadiabatic Holonomic Quantum Computation in Decoherence-Free Subspaces, Phys. Rev. Lett. 109, 170501 (2012).

[34] Z.-Y. Xue, J. Zhou, and Z. D. Wang, Universal holonomic quantum gates in decoherence-free subspace on superconducting circuits, Phys. Rev. A 92, 022320 (2015).

[35] Z.-Y. Xue, F.-L. Gu, Z.-P. Hong, Z.-H. Yang, D.-W. Zhang, Y. $\mathrm{Hu}$, and J. Q. You, Nonadiabatic Holonomic Quantum Computation with Dressed-State Qubits, Phys. Rev. Appl. 7, 054022 (2017).

[36] J. Zhou, B. J. Liu, Z. P. Hong, and Z. Y. Xue, Fast holonomic quantum computation based on solid-state spins with all-optical control, Sci. China-Phys. Mech. Astron. 61, 010312 (2017).

[37] Z.-P. Hong, B.-J. Liu, J.-Q. Cai, X.-D. Zhang, Y. Hu, Z. D. Wang, and Z.-Y. Xue, Implementing universal nonadiabatic holonomic quantum gates with transmons, Phys. Rev. A 97, 022332 (2018).

[38] V. Azimi Mousolou, Electric nonadiabatic geometric entangling gates on spin qubits, Phys. Rev. A 96, 012307 (2017).

[39] P. Z. Zhao, K. Z. Li, G. F. Xu, and D. M. Tong, General approach for constructing hamiltonians for nonadiabatic holonomic quantum computation, Phys. Rev. A 101, 062306 (2020).

[40] M. Johansson, E. Sjöqvist, L. M. Andersson, M. Ericsson, B. Hessmo, K. Singh, and D. M. Tong, Robustness of nonadiabatic holonomic gates, Phys. Rev. A 86, 062322 (2012).

[41] S.-B. Zheng, C.-P. Yang, and F. Nori, Comparison of the sensitivity to systematic errors between nonadiabatic non-Abelian geometric gates and their dynamical counterparts, Phys. Rev. A 93, 032313 (2016).

[42] N. Ramberg and E. Sjöqvist, Environment-Assisted Holonomic Quantum Maps, Phys. Rev. Lett. 122, 140501 (2019).

[43] J. Jing, C.-H. Lam, and L.-A. Wu, Non-Abelian holonomic transformation in the presence of classical noise, Phys. Rev. A 95, 012334 (2017).

[44] B.-J. Liu, Y.-S. Wang, and M.-H. Yung, Global property condition-based non-adiabatic geometric quantum control, arXiv:2008.02176.

[45] G. Feng, G. Xu, and G. Long, Experimental Realization of Nonadiabatic Holonomic Quantum Computation, Phys. Rev. Lett. 110, 190501 (2013).

[46] H. Li, Y. Liu, and G. L. Long, Experimental realization of single-shot nonadiabatic holonomic gates in nuclear spins, Sci. China-Phys. Mech. Astron. 60, 080311 (2017).

[47] A. A. Abdumalikov Jr, J. M. Fink, K. Juliusson, M. Pechal, S. Berger, A. Wallraff, and S. Filipp, Experimental realization of non-Abelian non-adiabatic geometric gates, Nature (London) 496, 482 (2013).

[48] Y. Xu, W. Cai, Y. Ma, X. Mu, L. Hu, T. Chen, H. Wang, Y. P. Song, Z.-Y. Xue, Z.-q. Yin, and L. Sun, Single-Loop Realization of Arbitrary Nonadiabatic Holonomic Single-Qubit Quantum Gates in a Superconducting Circuit, Phys. Rev. Lett. 121, 110501 (2018).

[49] Z. Zhang, P. Z. Zhao, T. Wang, L. Xiang, Z. Jia, P. Duan, D. M. Tong, Y. Yin, and G. Guo, Single-shot realization of nonadiabatic holonomic gates with a superconducting Xmon qutrit, New J. Phys. 21, 073024 (2019).

[50] S. Danilin, A. Vepsäläinen, and G. S. Paraoanu, Experimental state control by fast non-Abelian holonomic gates with a superconducting qutrit, Phys. Scr. 93, 055101 (2018). 
[51] D. J. Egger, M. Ganzhorn, G. Salis, A. Fuhrer, P. Müller, P. K. Barkoutsos, N. Moll, I. Tavernelli, and S. Filipp, Entanglement Generation in Superconducting Qubits Using Holonomic Operations, Phys. Rev. Appl. 11, 014017 (2019).

[52] C. Zu, W.-B. Wang, L. He, W.-G. Zhang, C.-Y. Dai, F. Wang, and L.-M. Duan, Experimental realization of universal geometric quantum gates with solid-state spins, Nature (London) 514, 72 (2014).

[53] S. Arroyo-Camejo, A. Lazariev, S. W. Hell, and G. Balasubramanian, Room temperature high-fidelity holonomic single-qubit gate on a solid-state spin, Nat. Commun. 5, 4870 (2014).

[54] B. B. Zhou, P. C. Jerger, V. O. Shkolnikov, F. J. Heremans, G. Burkard, and D. D. Awschalom, Holonomic Quantum Control by Coherent Optical Excitation in Diamond, Phys. Rev. Lett. 119, 140503 (2017).

[55] Y. Sekiguchi, N. Niikura, R. Kuroiwa, H. Kano, and H. Kosaka, Optical holonomic single quantum gates with a geometric spin under a zero field, Nat. Photonics 11, 309 (2017).

[56] K. Nagata, K. Kuramitani, Y. Sekiguchi, and H. Kosaka, Universal holonomic quantum gates over geometric spin qubits with polarised microwaves, Nat. Commun. 9, 3227 (2018).

[57] N. Ishida, T. Nakamura, T. Tanaka, S. Mishima, H. Kano, R. Kuroiwa, Y. Sekiguchi, and H. Kosaka, Universal holonomic single quantum gates over a geometric spin with phasemodulated polarized light, Opt. Lett. 43, 2380 (2018).

[58] S.-L. Zhu and Z. D. Wang, Unconventional Geometric Quantum Computation, Phys. Rev. Lett. 91, 187902 (2003).

[59] X.-L. Feng, C. Wu, H. Sun, and C. H. Oh, Geometric Entangling Gates in Decoherence-Free Subspaces with Minimal Requirements, Phys. Rev. Lett. 103, 200501 (2009).

[60] Y. Ota and Y. Kondo, Composite pulses in NMR as nonadiabatic geometric quantum gates, Phys. Rev. A 80, 024302 (2009).

[61] J. Spiegelberg and E. Sjöqvist, Validity of the rotating-wave approximation in nonadiabatic holonomic quantum computation, Phys. Rev. A 88, 054301 (2013).

[62] G. Xu and G. Long, Protecting geometric gates by dynamical decoupling, Phys. Rev. A 90, 022323 (2014)

[63] Z.-T. Liang, Y.-X. Du, W. Huang, Z.-Y. Xue, and H. Yan, Nonadiabatic holonomic quantum computation in decoherence-free subspaces with trapped ions, Phys. Rev. A 89, 062312 (2014).

[64] G. F. Xu, C. L. Liu, P. Z. Zhao, and D. M. Tong, Nonadiabatic holonomic gates realized by a single-shot implementation, Phys. Rev. A 92, 052302 (2015).

[65] V. V. Albert, C. Shu, S. Krastanov, C. Shen, R.-B. Liu, Z.-B. Yang, R. J. Schoelkopf, M. Mirrahimi, M. H. Devoret, and L. Jiang, Holonomic Quantum Control with Continuous Variable Systems, Phys. Rev. Lett. 116, 140502 (2016).

[66] P. Z. Zhao, G. F. Xu, and D. M. Tong, Nonadiabatic geometric quantum computation in decoherence-free subspaces based on unconventional geometric phases, Phys. Rev. A 94, 062327 (2016).

[67] P. Z. Zhao, G. F. Xu, Q. M. Ding, E. Sjöqvist, and D. M. Tong, Single-shot realization of nonadiabatic holonomic quantum gates in decoherence-free subspaces, Phys. Rev. A 95, 062310 (2017).

[68] P. Z. Zhao, X. Wu, T. H. Xing, G. F. Xu, and D. M. Tong, Nonadiabatic holonomic quantum computation with Rydberg superatoms, Phys. Rev. A 98, 032313 (2018).
[69] P. Z. Zhao, G. F. Xu, and D. M. Tong, Nonadiabatic holonomic multiqubit controlled gates, Phys. Rev. A 99, 052309 (2019).

[70] D. Leibfried, B. DeMarco, V. Meyer, D. Lucas, M. Barrett, J. Britton, W. M. Itano, B. Jelenković, C. Langer, T. Rosenband, and D. J. Wineland, Experimental demonstration of a robust, high-fidelity geometric two ion-qubit phase gate, Nature (London) 422, 412 (2003).

[71] J. Du, P. Zou, and Z. D. Wang, Experimental implementation of high-fidelity unconventional geometric quantum gates using an NMR interferometer, Phys. Rev. A 74, 020302(R) (2006).

[72] T. Yan, B.-J. Liu, K. Xu, C. Song, S. Liu, Z. Zhang, H Deng, Z. Yan, H. Rong, K. Huang, M.-H. Yung, Y. Chen, and D. Yu, Experimental Realization of Nonadiabatic Shortcut to Non-Abelian Geometric Gates, Phys. Rev. Lett. 122, 080501 (2019).

[73] Y. Xu, Z. Hua, T. Chen, X. Pan, X. Li, J. Han, W. Cai, Y. Ma, H. Wang, Y. P. Song, Z.-Y. Xue, and L. Sun, Experimental Implementation of Universal Nonadiabatic Geometric Quantum Gates in a Superconducting Circuit, Phys. Rev. Lett. 124, 230503 (2020).

[74] P. Z. Zhao, Z. Dong, Z. Zhang, G. Guo, D. M. Tong, and Y. Yin, Experimental realization of nonadiabatic geometric gates with a superconducting Xmon qubit, arXiv:1909.09970.

[75] U. Haeberlen and J. S. Waugh, Coherent averaging effects in magnetic resonance, Phys. Rev. 175, 453 (1968).

[76] L. M. K. Vandersypen and I. L. Chuang, NMR techniques for quantum control and computation, Rev. Mod. Phys. 76, 1037 (2005).

[77] N. Goldman and J. Dalibard, Periodically Driven Quantum Systems: Effective Hamiltonians and Engineered Gauge Fields, Phys. Rev. X 4, 031027 (2014).

[78] M. Bukov, L. D'Alessio, and A. Polkovnikov, Universal high-frequency behavior of periodically driven systems: from dynamical stabilization to Floquet engineering, Adv. Phys. 64, 139 (2015).

[79] A. Poudel, G. Ortiz, and L. Viola, Dynamical generation of Floquet Majorana flat bands in s-wave superconductors, Europhys. Lett. 110, 17004 (2015).

[80] A. Eckardt, Colloquium: Atomic quantum gases in periodically driven optical lattices, Rev. Mod. Phys. 89, 011004 (2017).

[81] T. Oka and S. Kitamura, Floquet engineering of quantum materials, Annu. Rev. Condens. Matter Phys. 10, 387 (2019).

[82] V. Novičenko, E. Anisimovas, and G. Juzeliūnas, Floquet analysis of a quantum system with modulated periodic driving, Phys. Rev. A 95, 023615 (2017).

[83] V. Novičenko and G. Juzeliūnas, Non-Abelian geometric phases in periodically driven systems, Phys. Rev. A 100, 012127 (2019).

[84] R. W. Bomantara and J. Gong, Quantum computation via Floquet topological edge modes, Phys. Rev. B 98, 165421 (2018).

[85] R. W. Bomantara and J. Gong, Simulation of Non-Abelian Braiding in Majorana Time Crystals, Phys. Rev. Lett. 120, 230405 (2018).

[86] B.-J. Liu, Z.-Y. Xue, and M.-H. Yung, Brachistochronic nonadiabatic holonomic quantum control, arXiv:2001.05182.

[87] S. Lloyd, Almost any Quantum Logic Gate is Universal, Phys. Rev. Lett. 75, 346 (1995). 
[88] M. Saffman, T. G. Walker, and K. Mølmer, Quantum information with Rydberg atoms, Rev. Mod. Phys. 82, 2313 (2010).

[89] M. Saffman, Quantum computing with atomic qubits and Rydberg interactions: progress and challenges, J. Phys. B 49, 202001 (2016).

[90] A. Omran, H. Levine, A. Keesling, G. Semeghini, T. T. Wang, S. Ebadi, H. Bernien, A. S. Zibrov, H. Pichler, S. Choi, J. Cui, M. Rossignolo, P. Rembold, S. Montangero, T. Calarco, M. Endres, M. Greiner, V. Vuletić, and M. D. Lukin, Generation and manipulation of Schrödinger cat states in Rydberg atom arrays, Science 365, 570 (2019).
[91] H. Bernien, S. Schwartz, A. Keesling, H. Levine, A. Omran, H. Pichler, S. Choi, A. S. Zibrov, M. Endres, M. Greiner, V. Vuletić, and M. D. Lukin, Probing many-body dynamics on a 51-atom quantum simulator, Nature (London) 551, 579 (2017).

[92] H. Levine, A. Keesling, A. Omran, H. Bernien, S. Schwartz, A. S. Zibrov, M. Endres, M. Greiner, V. Vuletić, and M. D. Lukin, High-Fidelity Control and Entanglement of RydbergAtom Qubits, Phys. Rev. Lett. 121, 123603 (2018).

[93] A. Somoroff, Q. Ficheux, R. A. Mencia, H. Xiong, R. V. Kuzmin, and V. E. Manucharyan, Millisecond coherence in a superconducting qubit, arXiv:2103.08578. 